\title{
Novel Stability Analysis for Uncertain Neutral-Type Lur'e Systems with Time-Varying Delays Using New Inequality
}

\author{
Yanmeng Wang, ${ }^{1}$ Lianglin Xiong, ${ }^{2,3}$ Yongkun Li, ${ }^{3}$ Haiyang Zhang, ${ }^{4}$ and Chen Peng ${ }^{2}$ \\ ${ }^{1}$ College of Sciences, Nanjing University of Aeronautics and Astronautics, Nanjing, China \\ ${ }^{2}$ Mathematics and Computer Science, Yunnan Minzu University, Kunming, China \\ ${ }^{3}$ School of Mathematics and Statistics, Yunnan University, Kunming, China \\ ${ }^{4}$ School of Science, Nanjing University of Science and Technology, Nanjing, China
}

Correspondence should be addressed to Lianglin Xiong; lianglin_5318@126.com

Received 8 October 2016; Revised 10 January 2017; Accepted 18 January 2017; Published 21 February 2017

Academic Editor: Sabri Arik

Copyright (c) 2017 Yanmeng Wang et al. This is an open access article distributed under the Creative Commons Attribution License, which permits unrestricted use, distribution, and reproduction in any medium, provided the original work is properly cited.

\begin{abstract}
This paper considers the delay-dependent stability analysis of neutral-type Lur'e systems with time-varying delays and sector bounded nonlinearities. First of all, using constructed function methods, a new Jensen-like inequality is introduced to obtain less conservative results. Second, a new class of Lyapunov-Krasovskii functional (LKF) is constructed according to the characteristic of the considered systems. Third, combining with the new inequality and reciprocal convex approach and some other inequality techniques, the new less conservative robust stability criteria are shown in the form of linear matrix inequalities (LMIs). Finally, three examples demonstrate the feasibility and the superiority of our methods.
\end{abstract}

\section{Introduction}

Delay phenomenon is often encountered in many practical systems, such as biological systems, chemical systems, electronic systems, and network control systems. However, time-delay is usually the main cause of instability and bad performance. Hence, many authors devote themselves to studying the stability and many effective methods of the timevarying system to gain less conservative delay-dependent stability criteria [1-11], which include linear systems with the delay-fraction theory $[2,3]$ and nonlinear systems Lur'e systems [6-8]. As is known to all, delay-dependent stability results are less conservative than the delay-independent ones if delay size is very small. Therefore, a lot of articles were published recently which studied the delay-dependent stability for a class of neutral-type Lure systems with timevarying delays and sector bounded nonlinearities, and lots of significant results have been developed [12-30].

Delay-dependent stability criteria were presented for nominal and uncertain neutral-type Lur'e systems with constant time delays and sector bounded nonlinearities in [27]. The robust stability problems for neutral-type Lur'e systems with time-varying delays were considered because time delays vary always depending on time in [12-21, 27-30]. The freeweighting matrix method was applied to get less conservative stability criteria and to deal with the robust stability problems for neutral-type Lur'e systems with time-varying delays in $[15,16]$. However, the free-weighting matrix method brings more variables which make the computation quite complex. So, it is the right time to improve the disadvantage of the freeweighting matrix method and to get less conservative stability criteria for neutral-type Lur'e systems with time-varying delays. Some new robust stability criteria were proposed without using the general free-weighting matrix method which are less conservative and easier to calculate than some previous ones in $[15,19]$. Reference [31] can reduce conservatism by reducing the conservatism of the Jensenlike inequality. Motivated by [31], developing the Jensenlike inequality with double-integral term may reduce the conservativeness. As a result, less conservative criteria may be also got by constructing new integral inequalities used in LKF.

This paper studies the stability for a class of neutral-type Lur'e systems with time-varying delays and sector bounded 
nonlinearities. To investigate the neutral-type Lur'e system, this paper introduces a new triple-integral inequality used in the following LKFs and gets less conservative criteria. The LKF contains not only double-integral terms but also triple-integral terms. Using some effective techniques, such as a novel integral inequality, a piecewise analysis method, and the reciprocally convex combination inequality, instead of the general free-weighting matrix method, the delaydependent stability criteria derived in the form of LMIs are less conservative than some existing results in other papers. The effectiveness and the less conservatism of stability criteria proposed in this paper are demonstrated by using numerical examples in Section 4.

Notation. $R^{n}$ denotes the $n$-dimensional Euclidean vector space, and $R^{n \times m}$ denotes the set of all $n \times m$ real matrices. For a symmetric matrix $P, P>0$ (resp., $P<0$ ) shows that $P$ is a positive (resp., negative) definite matrix. $\operatorname{diag}\left\{a_{1}, a_{2}, \ldots, a_{n}\right\}$ represents a diagonal matrix with diagonal elements $a_{1}, a_{2}, \ldots, a_{n}$. $*$ denotes a symmetric term in a symmetric matrix.

\section{Problem Statement and Preliminaries}

Consider a class of Lur'e systems of uncertain neutral type with time-varying delays and sector-bound nonlinearities described by the following equation:

$$
\begin{aligned}
\dot{x}(t)-C \dot{x}(t-\tau(t))= & {[A+\Delta A(t)] x(t) } \\
& +\left[A_{1}+\Delta A_{1}(t)\right] x(t-h(t)) \\
& +[B+\Delta B(t)] f(\sigma(t)), \\
\sigma(t)= & H^{T} x(t), \quad \forall t \geq 0, \\
x(s)= & \phi(s), \\
\dot{x}(s)= & \dot{\phi}(s),
\end{aligned}
$$

$$
s \in[-\max (h, \tau), 0],
$$

where $\sigma(t) \in R^{m}$ and $x(t) \in R^{n}$ stand for the output and state vectors, respectively. $\phi(s) \in R^{n}$ is a real-valued continuous initial function on $[-\max (h, \tau), 0] . A, A_{1}, B, C$, and $H$ are known real constant matrices with appropriate dimensions. $\Delta A(t), \Delta A_{1}(t)$, and $\Delta B(t)$ represent the time-varying uncertainty parameters. $f(\sigma(t)) \in R^{m}$ is the nonlinear function such as

$$
f(\sigma(t))=\left[\begin{array}{llll}
f_{1}\left(\sigma_{1}(t)\right) & f_{2}\left(\sigma_{2}(t)\right) & \cdots & f_{m}\left(\sigma_{m}(t)\right)
\end{array}\right]^{T},
$$

where $\sigma_{i}(t)$ is the $i$ th component of the output vector $\sigma(t)$, and each term $f_{i}\left(\sigma_{i}(t)\right)(i=1,2, \ldots, m)$ satisfies the finite sector condition shown in Figure 1(a) [32]

$$
\begin{gathered}
f_{i}\left(\sigma_{i}(t)\right) \in K_{\left[0, k_{i}\right]}=\left\{f_{i}\left(\sigma_{i}(t)\right) \mid f_{i}(0)=0,0<\sigma_{i}(t)\right. \\
\left.\cdot f_{i}\left(\sigma_{i}(t)\right) \leq k_{i} \sigma_{i}^{2}(t), \sigma_{i}(t) \neq 0\right\}
\end{gathered}
$$

with known positive scalars $k_{i}$ or the infinite sector condition shown in Figure 1(b) [32]

$$
\begin{gathered}
f_{i}\left(\sigma_{i}(t)\right) \in K_{[0, \infty]}=\left\{f_{i}\left(\sigma_{i}(t)\right) \mid f_{i}(0)=0, \sigma_{i}(t)\right. \\
\left.\cdot f_{i}\left(\sigma_{i}(t)\right)>0, \sigma_{i}(t) \neq 0\right\} .
\end{gathered}
$$

$\Delta A(t), \Delta A_{1}(t)$, and $\Delta B(t)$ are assumed to satisfy the following condition:

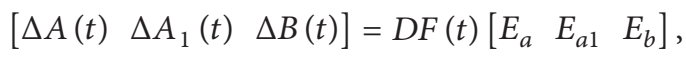

where $D, E_{a}, E_{a 1}$, and $E_{b}$ are known constant matrices with appropriate dimensions, and the uncertainty time-varying matrix $F(t)$ satisfies

$$
F^{T}(t) F(t) \leq I, \quad \forall t \geq 0
$$

The time-varying delays $h(t)$ and $\tau(t)$ are continuous functions satisfying the following conditions:

$$
\begin{aligned}
0 & \leq h(t) \leq h, \\
\dot{h}(t) & \leq h_{d}, \\
0 & \leq \tau(t) \leq \tau, \\
\dot{\tau}(t) & \leq \tau_{d}<1,
\end{aligned}
$$

$$
\forall t \geq 0
$$

This paper investigates the delay-dependent stability of Lur'e system (1) satisfying conditions (3) (or (4)) and (5)-(7) to gain less conservative robust stability criteria by using a new inequality and constructing a new LKF. Throughout this paper, the results will be acquired by assuming that all the eigenvalues of $C$ are inside the unit circle [33]. The following lemmas are useful in deriving criteria.

Lemma 1 (see [34] (reciprocally convex combination)). Let $f_{1}, f_{2}, \ldots, f_{N}: R^{m} \mapsto R$ have positive values in an open subset $D$ of $R^{m}$. Then, the reciprocally convex combination of $f_{i}$ over $D$ satisfies

$$
\begin{aligned}
\min _{\left\{\alpha_{i} \mid \alpha_{i}>0, \sum_{i} \alpha_{i}=1\right\}} & \sum_{i} \frac{1}{\alpha_{i}} f_{i}(t)=\sum_{i} f_{i}(t)+\max _{g_{i, j}(t)} \sum_{i \neq j} g_{i, j}(t) \\
\text { subject to } & \left\{g_{i, j}: R^{m} \longmapsto R, g_{j, i}(t) \triangleq g_{i, j}(t),\left[\begin{array}{cc}
f_{i}(t) & g_{i, j}(t) \\
g_{i, j}(t) & f_{j}(t)
\end{array}\right] \geq 0\right\} .
\end{aligned}
$$




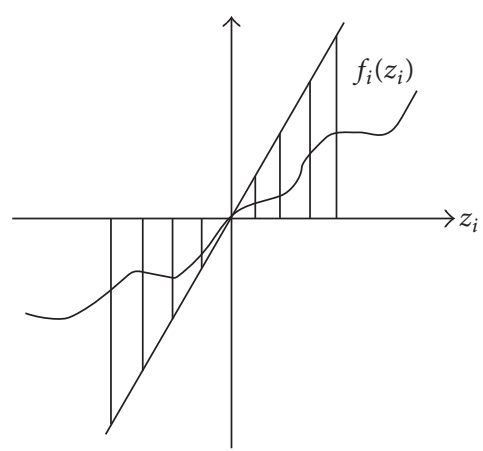

(a) Finite sector bounded nonlinearity

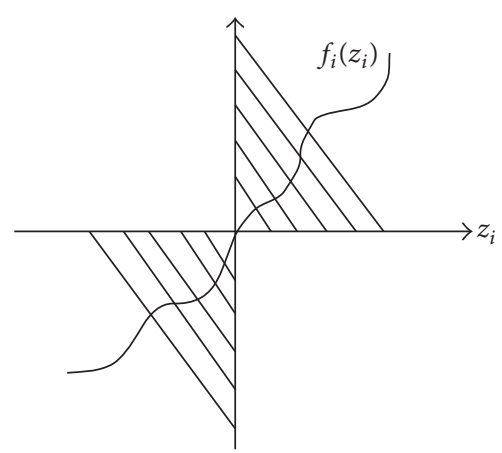

(b) Infinite sector bounded nonlinearity

FIGURE 1: Two sector bounded nonlinearity.

Lemma 2 (see [31]). For a given matrix $R>0$, any differentiable function $\omega$ in $[a, b] \rightarrow R^{n}$, the following inequality holds:

$$
\begin{aligned}
\int_{a}^{b} \dot{\omega}^{T}(u) R \dot{\omega}(u) d u \geq & \frac{1}{b-a}(\omega(b)-\omega(a))^{T} R \\
& \cdot(\omega(b)-\omega(a)) \\
& +\frac{3}{b-a} \Omega_{1}^{T} R \Omega_{1},
\end{aligned}
$$

where

$$
\Omega_{1}=\omega(b)+\omega(a)-\frac{2}{b-a} \int_{a}^{b} \omega(u) d u .
$$

Remark 3. It is clear that this new inequality encompasses the Jensen inequality. It is also worth noting that it plays an important role in getting the derivative of the LKF. Hence, by using optimization theory and improving the Jensen-like inequality, the double-integral inequality would be got in the following lemma.

Lemma 4. For any matrix $R>0$, scalars $h_{2} \geq h_{1} \geq 0$, and any continuous function $\omega$ in $R \rightarrow R^{n}$ the following inequality holds:

$$
\begin{aligned}
- & \int_{-h_{2}}^{-h_{1}} \int_{t+\theta}^{t} \omega^{T}(u) R \omega(u) d u d \theta \\
\leq & -\frac{2}{h_{2}^{2}-h_{1}^{2}}\left(\int_{-h_{2}}^{-h_{1}} \int_{t+\theta}^{t} \omega(u) d u d \theta\right)^{T} R \\
& \cdot\left(\int_{-h_{2}}^{-h_{1}} \int_{t+\theta}^{t} \omega(u) d u d \theta\right)-\frac{48}{h_{2}^{4}-h_{1}^{4}} \Omega_{2}^{T} R \Omega_{2},
\end{aligned}
$$

where

$$
\begin{aligned}
\Omega_{2}= & \frac{h_{1}}{2} \int_{-h_{2}}^{-h_{1}} \int_{t+\theta}^{t} \omega(u) d u d \theta \\
& +\frac{1}{2} \int_{-h_{2}}^{-h_{1}} \int_{-h_{2}}^{\theta} \int_{t+r}^{t} \omega(u) d u d r d \theta \\
& -\int_{-h_{2}}^{-h_{1}} \int_{t+\theta}^{t} \int_{t+\theta}^{u} \omega(s) d s d u d \theta
\end{aligned}
$$

Proof. Recall that the objectives of the present paper lemma are to acquire new lower bounds of the integral $\int_{-h_{2}}^{-h_{1}} \int_{t+\theta}^{t} \omega^{T}(u) R \omega(u) d u d \theta$ and to compare with the Jensenlike inequality. For this reason, the appropriate function $z(u)$ is given as the following form:

$$
\begin{aligned}
z(u)= & \omega(u)-\frac{2}{h_{2}^{2}-h_{1}^{2}} \int_{-h_{2}}^{-h_{1}} \int_{t+\theta}^{t} \omega(u) d u d \theta \\
& -\frac{48 f(u, \theta)}{h_{2}^{4}-h_{1}^{4}} \Omega_{2},
\end{aligned}
$$

where $f(u, \theta)=u-(1 / 2) \theta-t$ is constructed to satisfy

$$
\int_{-h_{2}}^{-h_{1}} \int_{t+\theta}^{t} f(u, \theta) d u d \theta=0
$$

and to get rid of the effect of cross terms. It is easy to obtain

$$
\begin{aligned}
& \int_{-h_{2}}^{-h_{1}} \int_{t+\theta}^{t} f^{2}(u, \theta) d u d \theta=\frac{h_{2}^{4}-h_{1}^{4}}{48} \\
& \int_{-h_{2}}^{-h_{1}} \int_{t+\theta}^{t} \omega(u) f(u, \theta) d u d \theta \\
& =\frac{h_{1}}{2} \int_{-h_{2}}^{-h_{1}} \int_{t+\theta}^{t} \omega(u) d u d \theta \\
& \quad-\int_{-h_{2}}^{-h_{1}} \int_{t+\theta}^{t} \omega(u)(t-u) d u d \theta \\
& \quad-\frac{1}{2} \int_{-h_{2}}^{-h_{1}} \int_{t+\theta}^{t} \omega(u)\left(h_{1}+\theta\right) d u d \theta \\
& =\frac{h_{1}}{2} \int_{-h_{2}}^{-h_{1}} \int_{t+\theta}^{t} \omega(u) d u d \theta \\
& \quad+\frac{1}{2} \int_{-h_{2}}^{-h_{1}} \int_{-h_{2}}^{\theta} \int_{t+r}^{t} \omega(u) d u d r d \theta \\
& \quad-\int_{-h_{2}}^{-h_{1}} \int_{t+\theta}^{t} \int_{t+\theta}^{u} \omega(s) d s d u d \theta=\Omega_{2} .
\end{aligned}
$$


Then, based on the formulas above, the following equality holds:

$$
\begin{aligned}
& \int_{-h_{2}}^{-h_{1}} \int_{t+\theta}^{t} z^{T}(u) R z(u) d u d \theta \\
& =\int_{-h_{2}}^{-h_{1}} \int_{t+\theta}^{t} \omega^{T}(u) R \omega(u) d u d \theta \\
& -\frac{2}{h_{2}^{2}-h_{1}^{2}}\left(\int_{-h_{2}}^{-h_{1}} \int_{t+\theta}^{t} \omega(u) d u d \theta\right)^{T} \\
& \cdot R\left(\int_{-h_{2}}^{-h_{1}} \int_{t+\theta}^{t} \omega(u) d u d \theta\right)+\frac{48^{2}}{\left(h_{2}^{4}-h_{1}^{4}\right)^{2}} \\
& \cdot \int_{-h_{2}}^{-h_{1}} \int_{t+\theta}^{t} f^{2}(u, \theta) d u d \theta \Omega_{2}^{T} R \Omega_{2} \\
& -\frac{2 \cdot 48}{h_{2}^{4}-h_{1}^{4}}\left(\int_{-h_{2}}^{-h_{1}} \int_{t+\theta}^{t} \omega(u) f(u, \theta) d u d \theta\right)^{T} R \Omega_{2} \\
& +\frac{4 \cdot 48}{\left(h_{2}^{4}-h_{1}^{4}\right)\left(h_{2}^{2}-h_{1}^{2}\right)} \int_{-h_{2}}^{-h_{1}} \int_{t+\theta}^{t} f(u, \theta) d u d \theta \\
& \cdot\left(\int_{-h_{2}}^{-h_{1}} \int_{t+\theta}^{t} \omega(u) d u d \theta\right)^{T} R \Omega_{2} \\
& =\int_{-h_{2}}^{-h_{1}} \int_{t+\theta}^{t} \omega^{T}(u) R \omega(u) d u d \theta \\
& -\frac{2}{h_{2}^{2}-h_{1}^{2}}\left(\int_{-h_{2}}^{-h_{1}} \int_{t+\theta}^{t} \omega(u) d u d \theta\right)^{T} \\
& \cdot R\left(\int_{-h_{2}}^{-h_{1}} \int_{t+\theta}^{t} \omega(u) d u d \theta\right)-\frac{48}{h_{2}^{4}-h_{1}^{4}} \Omega_{2}^{T} R \Omega_{2} \text {. }
\end{aligned}
$$

For a given symmetric positive definite matrix $R>0$, then, the left-hand side of formula (16) above is positive definite. It is obvious that Lemma 4 is correct.

Remark 5. Compared with Lemma 1 in [35], the inequality in Lemma 4 has two advantages. First, since $R>0$, the second term in the right side of the inequality in Lemma 4 is definite negative. It thus implies that the Jensen-like inequality in [35] has been included in the inequality proposed above. Second, it is also worth noting that this improvement is allowed by using extra signals $\int_{-h_{2}}^{-h_{1}} \int_{-h_{2}}^{\theta} \int_{t+r}^{t} \omega(u) d u d r d \theta, \int_{-h_{2}}^{-h_{1}} \int_{t+\theta}^{t} \int_{t+\theta}^{u} \omega(s) d s d u d \theta$, and $\int_{-h_{2}}^{-h_{1}} \int_{t+\theta}^{t} \omega(u) d u d \theta$.

Remark 6. It is worth paying attention to the fact that the construction of function $f$ is the key to Lemma 4 . $\int_{-h}^{0} \int_{t+\theta}^{t} f d u d \theta=0$ must be sure when we construct the function $f$ to get rid of crossing items and to make the second term in the right side of our inequality be definite negative.
Remark 7. In fact, the result of Lemma 4 is less conservative than formula (3) in literature [36]. When $h_{1} \neq 0$, the result of Lemma 4 is different from inequality (16) in literature [37]. What is more, under the special scaling condition of the left side of the inequality in Lemma 4, the more conservativeness of inequality (16) in literature [37] would be easily concluded. To some extent, the conditions of Lemma 4 are wider than inequality (16) in literature [37] in terms of integral domain. Actually, with the help of delay decomposition technology, $\int_{-h / 2}^{0} \int_{t+\theta}^{t} f(s) d s d \theta$ and $\int_{-h}^{-h / 2} \int_{t+\theta}^{t} f(s) d s d \theta$ can be scaled properly with our inequality in Lemma 4. In our future research, we will try to use formulas in the literature [37] which may be more flexible than the Lemma 4 to reduce the conservatism of stability criteria in another case.

\section{Main Results}

To accept easily the robust stability problem of system (1), firstly, we investigate the stability criterion of the nominal form:

$$
\begin{aligned}
& \dot{x}(t)-C \dot{x}(t-\tau(t))= A x(t)+A_{1} x(t-h(t)) \\
&+B f(\sigma(t)), \\
& \sigma(t)= H^{T} x(t), \quad \forall t \geq 0, \\
& x(s)= \phi(s), \\
& \dot{x}(s)=\dot{\phi}(s),
\end{aligned}
$$

The stability of system (17) can be analyzed via timeindependent functions of the form

$$
V(x(t))=\sum_{i=1}^{5} V_{i}(x(t)),
$$

where

$$
\begin{aligned}
& V_{1}(x(t))=\xi_{1}^{T}(t) P \xi_{1}(t)+2 \sum_{i=1}^{m} \lambda_{i} \int_{0}^{\sigma_{i}(t)} f_{i}(\sigma) d \sigma \\
& V_{2}(x(t))=\int_{t-h / 2}^{t}\left[\begin{array}{c}
x(s) \\
x\left(s-\frac{h}{2}\right)
\end{array}\right] \\
& \cdot\left[\begin{array}{cc}
X_{11} & X_{12} \\
* & X_{22}
\end{array}\right]\left[\begin{array}{c}
x(s) \\
x\left(s-\frac{h}{2}\right)
\end{array}\right] d s \\
& V_{3}(x(t))=\int_{t-h(t)}^{t} x^{T}(s) Q_{1} x(s) d s+\int_{t-\tau(t)}^{t} \dot{x}^{T}(s) \\
& \cdot Q_{2} \dot{x}(s) d s
\end{aligned}
$$




$$
\begin{aligned}
& V_{4}(x(t))=\frac{h^{2}}{8} \\
& \quad \cdot \int_{-h / 2}^{0} \int_{t+\theta}^{t}(s-t-\theta) \dot{x}^{T}(s) Q_{3} \dot{x}(s) d s d \theta+\frac{3 h^{2}}{8} \\
& \quad \cdot \int_{-h}^{-h / 2} \int_{t+\theta}^{t}(s-t-\theta) \dot{x}^{T}(s) Q_{4} \dot{x}(s) d s d \theta
\end{aligned}
$$

$$
\begin{gathered}
V_{5}(x(t))=\frac{h}{2} \int_{-h / 2}^{0} \int_{t+\theta}^{t} \dot{x}^{T}(s) Q_{5} \dot{x}(s) d s d \theta+\frac{h}{2} \\
\cdot \int_{-h}^{-h / 2} \int_{t+\theta}^{t} \dot{x}^{T}(s) Q_{6} \dot{x}(s) d s d \theta,
\end{gathered}
$$

and define

$$
\begin{aligned}
& \xi_{1}^{T}(t)=\left[x^{T}(t) \int_{t-h / 2}^{t} x^{T}(s) d s \int_{t-h}^{t-h / 2} x^{T}(s) d s \int_{-h / 2}^{0} \int_{t+\theta}^{t} x^{T}(s) d s d \theta \int_{-h}^{-h / 2} \int_{t+\theta}^{t} x^{T}(s) d s d \theta\right] \\
& \xi^{T}(t) \\
& =\left[\begin{array}{lllll}
x^{T}(t) & x^{T}(t-h(t)) & x^{T}\left(t-\frac{h}{2}\right) x^{T}(t-h) & \dot{x}^{T}(t-\tau(t)) & f^{T}(\sigma(t)) \int_{t-h / 2}^{t} x^{T}(s) d s \int_{t-h}^{t-h / 2} x^{T}(s) d s \int_{-h / 2}^{0} \int_{t+\theta}^{t} x^{T}(s) d s d \theta \int_{-h}^{-h / 2} \int_{t+\theta}^{t} x^{T}(s) d s d \theta
\end{array}\right] .
\end{aligned}
$$

Remark 8. It is worth noticing that the construction of LKF can be improved by adding

$$
\begin{aligned}
& \frac{h}{2} \int_{-h / 2}^{0} \int_{t+\theta}^{t} x^{T}(s) N_{1} x(s) d s d \theta \\
& \frac{h}{2} \int_{-h}^{-h / 2} \int_{t+\theta}^{t} x^{T}(s) N_{2} x(s) d s d \theta
\end{aligned}
$$

to get less conservative stability criteria for any positive definite matrices $N_{1}$ and $N_{2}$. However, our methods have reduced the conservatism of the existing results in the instance. Hence, this paper dose not add $N_{1}$ and $N_{2}$ to control the number of variables.

Remark 9. A new Lyapunov-Krasovskii functional is constructed in Section 3 to obtain a new delay-dependent stability criterion, which includes the relationship among the states, the nonlinear function, and the derivative of the states. In order to fully reduce the conservative of the condition, the constant delay $h$ is decomposed into $h / 2$ and thus (18) is obtained. Particularly, the function $V_{1}$ is constructed to eliminate the effects among different forms of the states; the function $V_{2}$ is constructed to eliminate the relationship among the states with different delays; to consider the time-varying delay the function $V_{3}$ is constructed; and our Lyapunov-Krasovskii functional contains double-integral (function $V_{4}$ ) and tripleintegral (function $V_{5}$ ) terms which yield less conservative delay-dependent stability criteria. Therefore, more information for the state is employed in constructing the more general Lyapunov-Krasovskii functionals, which may lead to reduced conservatism.

If nominal system (17) satisfies conditions (3) and (7) the following theorem can be got.

Theorem 10. Nominal system (17) satisfying conditions (3) and (7) is asymptotically stable for given values $h \geq 0$, $h_{d}, \tau_{d}<1$, and $k_{l}>0(l=1,2, \ldots, m)$, if there exist appropriate dimensional matrices $X_{1}, X_{2}, X_{12}$, positive matrices $P=\left[P_{i j}\right]_{5 \times 5}, Q_{i}(i=1,2, \ldots, 6), X_{11}, X_{22}$ positive semidefinite diagonal matrices $S=\operatorname{diag}\left\{s_{1}, s_{2}, \ldots, s_{m}\right\}$, and $\Lambda=\operatorname{diag}\left\{\lambda_{1}, \lambda_{2}, \ldots, \lambda_{m}\right\}$ such that the following LMIs hold:

$$
\begin{aligned}
& {\left[\begin{array}{ll}
Q_{5} & X_{1} \\
* & Q_{5}
\end{array}\right]>0,} \\
& {\left[\begin{array}{cc}
Q_{6} & X_{2} \\
* & Q_{6}
\end{array}\right]>0,} \\
& {\left[\begin{array}{cc}
X_{11} & X_{12} \\
* & X_{22}
\end{array}\right]>0,} \\
& \Xi_{r}=\left[\begin{array}{cc}
E+\Theta_{1}+\Psi_{r} & \eta^{T} \Omega \\
* & -\Omega
\end{array}\right]<0, \quad r=1,2,
\end{aligned}
$$

where

$$
\begin{aligned}
E= & {\left[E_{i j}\right]_{10 \times 10}, \quad E_{i j}=E_{j i}, i, j=1,2, \ldots, 10, } \\
E_{11}= & P_{11} A+P_{12}+\frac{1}{2} h P_{14}+\frac{1}{2} h P_{15}+A^{T} P_{11}+P_{12}^{T} \\
& +\frac{1}{2} h P_{14}^{T}+\frac{1}{2} h P_{15}^{T}+X_{11}+Q_{1}-\frac{37}{40} h^{2} Q_{4} \\
& -\frac{5}{8} h^{2} Q_{3}, \\
E_{12}= & P_{11} A_{1}, \\
E_{13}= & -P_{12}+P_{13}+X_{12}, \\
E_{14}= & -P_{13}, \\
E_{15}= & P_{11} C, \\
E_{16}= & P_{11} B+A^{T} H \Lambda, \\
E_{17}= & -P_{14}+A^{T} P_{12}+P_{22}+\frac{h}{2} P_{24}^{T}+\frac{h}{2} P_{25}^{T}-\frac{9 h}{10} Q_{4} \\
& -h Q_{3},
\end{aligned}
$$




$$
\begin{aligned}
& E_{18}=-P_{15}+A^{T} P_{13}+P_{23}+\frac{1}{2} h P_{34}^{T}+\frac{1}{2} h P_{35}^{T} \\
& E_{68}=B^{T} P_{13} \text {, } \\
& -\frac{13}{10} h Q_{4} \\
& E_{19}=A^{T} P_{14}+P_{24}+\frac{1}{2} h P_{44}+\frac{1}{2} h P_{45}^{T}+9 Q_{3} \text {, } \\
& E_{1,10}=A^{T} P_{15}+P_{25}+\frac{1}{2} h P_{45}+\frac{1}{2} h P_{55}+\frac{27}{5} Q_{4} \text {, } \\
& E_{22}=-\left(1-h_{d}\right) Q_{1} \text {, } \\
& E_{26}=A_{1}^{T} H \Lambda \text {, } \\
& E_{27}=A_{1}^{T} P_{12} \text {, } \\
& E_{28}=A_{1}^{T} P_{13} \text {, } \\
& E_{29}=A_{1}^{T} P_{14}, \\
& E_{2,10}=A_{1}^{T} P_{15} \\
& E_{33}=X_{22}-X_{11} \text {, } \\
& E_{34}=-X_{12} \text {, } \\
& E_{37}=-P_{22}+P_{23}^{T}, \\
& E_{38}=-P_{23}+P_{33} \text {, } \\
& E_{39}=-P_{24}+P_{34} \text {, } \\
& E_{3,10}=-P_{25}+P_{35} \text {, } \\
& E_{44}=-X_{22} \text {, } \\
& E_{47}=-P_{23}^{T} \text {, } \\
& E_{48}=-P_{33} \text {, } \\
& E_{49}=-P_{34}, \\
& E_{4,10}=-P_{35} \text {, } \\
& E_{55}=-\left(1-\tau_{d}\right) Q_{2}, \\
& E_{56}=C^{T} H \Lambda \text {, } \\
& E_{57}=C^{T} P_{12} \text {, } \\
& E_{58}=C^{T} P_{13} \text {, } \\
& E_{59}=C^{T} P_{14} \text {, } \\
& E_{5,10}=C^{T} P_{15} \text {, } \\
& E_{66}=\Lambda H^{T} B+B^{T} H \Lambda \text {, } \\
& E_{67}=B^{T} P_{12} \text {, } \\
& E_{69}=B^{T} P_{14}, \\
& E_{6,10}=B^{T} P_{15} \text {, } \\
& E_{77}=-P_{24}-P_{24}^{T}-\frac{6}{5} Q_{4}-7 Q_{3}, \\
& E_{78}=-P_{25}-P_{34}^{T}-\frac{12}{5} Q_{4}, \\
& E_{79}=-P_{44}+\frac{36}{h} Q_{3}, \\
& E_{7,10}=-P_{45}+\frac{36}{5 h} Q_{4} \text {, } \\
& E_{88}=-P_{35}-P_{35}^{T}-\frac{29}{5} Q_{4} \text {, } \\
& E_{89}=-P_{45}^{T}, \\
& E_{8,10}=-P_{55}+\frac{72}{5 h} Q_{4} \text {, } \\
& E_{99}=-\frac{216}{h^{2}} Q_{3} \text {, } \\
& E_{10,10}=-\frac{216}{5 h^{2}} Q_{4} \\
& E_{23}=E_{24}=E_{25}=E_{35}=E_{36}=E_{45}=E_{46}=E_{9,10} \\
& =0 \text {, } \\
& \Psi_{1}=-\left(e_{3}-e_{4}\right)^{T} Q_{6}\left(e_{3}-e_{4}\right) \\
& -3\left(e_{3}+e_{4}-\frac{4}{h} e_{8}\right)^{T} Q_{6} \cdot\left(e_{3}+e_{4}-\frac{4}{h} e_{8}\right) \\
& -\left[\begin{array}{c}
e_{2}-e_{3} \\
e_{1}-e_{2}
\end{array}\right]^{T}\left[\begin{array}{cc}
Q_{5} & X_{1} \\
* & Q_{5}
\end{array}\right]\left[\begin{array}{c}
e_{2}-e_{3} \\
e_{1}-e_{2}
\end{array}\right], \\
& \Psi_{2}=-\left(e_{1}-e_{3}\right)^{T} Q_{5}\left(e_{1}-e_{3}\right) \\
& -3\left(e_{1}+e_{3}-\frac{4}{h} e_{7}\right)^{T} Q_{5} \cdot\left(e_{1}+e_{3}-\frac{4}{h} e_{7}\right) \\
& -\left[\begin{array}{c}
e_{2}-e_{4} \\
e_{3}-e_{2}
\end{array}\right]^{T}\left[\begin{array}{cc}
Q_{6} & X_{2} \\
* & Q_{6}
\end{array}\right]\left[\begin{array}{l}
e_{2}-e_{4} \\
e_{3}-e_{2}
\end{array}\right], \\
& \Theta_{1}=e_{1}^{T} \mathrm{HKSe}_{6}-e_{6}^{T} \mathrm{Se}_{6}+\left(e_{1}^{T} \mathrm{HKS}_{6}-e_{6}^{T} S e_{6}\right)^{T} \text {, } \\
& e_{i}=\left[\begin{array}{lllllll}
0 & \cdots & 0 & \underbrace{1}_{i} & 0 & \cdots & 0
\end{array}\right]_{1 \times 10}, \\
& \eta=\left[\begin{array}{llllllllll}
A & A_{1} & 0 & 0 & C & B & 0 & 0 & 0 & 0
\end{array}\right] \text {, } \\
& \Omega=Q_{2}+\frac{h^{4}}{64} Q_{3}+\frac{9 h^{4}}{64} Q_{4}+\frac{h^{2}}{4} Q_{5}+\frac{h^{2}}{4} Q_{6} .
\end{aligned}
$$


Proof. The time-derivative of functions $V_{i}(x(t))$ can be calculated, respectively, based on the trajectory of system (17) as follows:

$$
\begin{aligned}
& \dot{V}_{1}(x(t))=2 \xi_{1}^{T} P \dot{\xi}_{1}+2 f^{T}(\sigma(t)) \Lambda H^{T} \dot{x}(t) \\
& \dot{V}_{2}(x(t)) \\
& =\left[\begin{array}{c}
x(t) \\
x\left(t-\frac{h}{2}\right)
\end{array}\right]^{T}\left[\begin{array}{cc}
X_{11} & X_{12} \\
* & X_{22}
\end{array}\right]\left[\begin{array}{c}
x(t) \\
x\left(t-\frac{h}{2}\right)
\end{array}\right] \\
& -\left[\begin{array}{c}
x\left(t-\frac{h}{2}\right) \\
x(t-h)
\end{array}\right]^{T}\left[\begin{array}{cc}
X_{11} & X_{12} \\
* & X_{22}
\end{array}\right]\left[\begin{array}{c}
x\left(t-\frac{h}{2}\right) \\
x(t-h)
\end{array}\right] \\
& \dot{V}_{3}(x(t)) \\
& \leq x^{T}(t) Q_{1} x(t)-\left(1-h_{d}\right) x^{T}(t-h(t)) Q_{1} \\
& \cdot x(t-h(t))+\dot{x}^{T}(t) Q_{2} \dot{x}(t) \\
& -\left(1-\tau_{d}\right) \dot{x}^{T}(t-\tau(t)) Q_{2} \dot{x}(t-\tau(t)) \\
& \dot{V}_{4}(x(t)) \\
& =\dot{x}^{T}(t)\left(\frac{h^{4}}{64} Q_{3}+\frac{9 h^{4}}{64} Q_{4}\right) \dot{x}(t) \\
& -\frac{h^{2}}{8} \int_{-h / 2}^{0} \int_{t+\theta}^{t} \dot{x}^{T}(s) Q_{3} \dot{x}(s) d s d \theta \\
& -\frac{3 h^{2}}{8} \int_{-h}^{-h / 2} \int_{t+\theta}^{t} \dot{x}^{T}(s) Q_{4} \dot{x}(s) d s d \theta \\
& \dot{V}_{5}(x(t)) \\
& =\frac{h^{2}}{4} \dot{x}^{T}(t)\left(Q_{5}+Q_{6}\right) \dot{x}(t) \\
& -\frac{h}{2} \int_{t-h / 2}^{t} \dot{x}^{T}(s) Q_{5} \dot{x}(s) d s \\
& -\frac{h}{2} \int_{t-h}^{t-h / 2} \dot{x}^{T}(s) Q_{6} \dot{x}(s) d s .
\end{aligned}
$$

Using Lemma 4 and the method of the exchange of integral order, we get

$$
\begin{aligned}
- & \frac{h^{2}}{8} \int_{-h / 2}^{0} \int_{t+\theta}^{t} \dot{x}^{T}(s) Q_{3} \dot{x}(s) d s d \theta \\
\leq & -\left(\int_{-h / 2}^{0} \int_{t+\theta}^{t} \dot{x}(s) d s d \theta\right)^{T} \\
\cdot & Q_{3}\left(\int_{-h / 2}^{0} \int_{t+\theta}^{t} \dot{x}(s) d s d \theta\right) \\
& -\frac{96}{h^{2}}\left(\frac{1}{2} \int_{-h / 2}^{0} \int_{-h / 2}^{\theta} \int_{t+r}^{t} \dot{x}(s) d s d r d \theta\right.
\end{aligned}
$$

$\left.-\int_{-h / 2}^{0} \int_{t+\theta}^{t} \int_{t+\theta}^{u} \dot{x}(s) d s d u d \theta\right)^{T}$

$\cdot Q_{3}\left(\frac{1}{2} \int_{-h / 2}^{0} \int_{-h / 2}^{\theta} \int_{t+r}^{t} \dot{x}(s) d s d r d \theta\right.$

$\left.-\int_{-h / 2}^{0} \int_{t+\theta}^{t} \int_{t+\theta}^{u} \dot{x}(s) d s d u d \theta\right)=-\left(\frac{h}{2} x(t)\right.$

$\left.-\int_{t-h / 2}^{t} x(s) d s\right)^{T} Q_{3}\left(\frac{h}{2} x(t)-\int_{t-h / 2}^{t} x(s) d s\right)$

$-\frac{96}{h^{2}}\left(\frac{h^{2}}{16} x(t)+\frac{h}{4} \int_{t-h / 2}^{t} x(s) d s\right.$

$\left.-\frac{3}{2} \int_{-h / 2}^{0} \int_{t+\theta}^{t} x(s) d s d \theta\right)^{T} \cdot Q_{3}\left(\frac{h^{2}}{16} x(t)\right.$

$\left.+\frac{h}{4} \int_{t-h / 2}^{t} x(s) d s-\frac{3}{2} \int_{-h / 2}^{0} \int_{t+\theta}^{t} x(s) d s d \theta\right)$

$-\frac{3 h^{2}}{8} \int_{-h}^{-h / 2} \int_{t+\theta}^{t} \dot{x}^{T}(s) Q_{4} \dot{x}(s) d s d \theta$

$\leq-\left(\int_{-h}^{-h / 2} \int_{t+\theta}^{t} \dot{x}(s) d s d \theta\right)^{T}$

$\cdot Q_{4}\left(\int_{-h}^{-h / 2} \int_{t+\theta}^{t} \dot{x}(s) d s d \theta\right)$

$-\frac{96}{5 h^{2}}\left(\frac{h}{4} \int_{-h}^{-h / 2} \int_{t+\theta}^{t} \dot{x}(s) d s d \theta\right.$

$+\frac{1}{2} \int_{-h}^{-h / 2} \int_{-h}^{\theta} \int_{t+r}^{t} \dot{x}(s) d s d r d \theta$

$\left.-\int_{-h}^{-h / 2} \int_{t+\theta}^{t} \int_{t+\theta}^{u} \dot{x}(s) d s d u d \theta\right)^{T}$

$\cdot Q_{4}\left(\frac{h}{4} \int_{-h}^{-h / 2} \int_{t+\theta}^{t} \dot{x}(s) d s d \theta\right.$

$+\frac{1}{2} \int_{-h}^{-h / 2} \int_{-h}^{\theta} \int_{t+r}^{t} \dot{x}(s) d s d r d \theta$

$\left.-\int_{-h}^{-h / 2} \int_{t+\theta}^{t} \int_{t+\theta}^{u} \dot{x}(s) d s d u d \theta\right)=-\left(\frac{h}{2} x(t)\right.$

$\left.-\int_{t-h}^{t-h / 2} x(s) d s\right)^{T} Q_{4}\left(\frac{h}{2} x(t)-\int_{t-h}^{t-h / 2} x(s) d s\right)$

$-\frac{96}{5 h^{2}}\left(\frac{3 h^{2}}{16} x(t)+\frac{h}{4} \int_{t-h / 2}^{t} x(s) d s\right.$

$\left.+\frac{h}{2} \int_{t-h}^{t-h / 2} x(s) d s-\frac{3}{2} \int_{-h}^{-h / 2} \int_{t+\theta}^{t} x(s) d s d \theta\right)^{T}$ 


$$
\begin{aligned}
& \cdot Q_{4}\left(\frac{3 h^{2}}{16} x(t)+\frac{h}{4} \int_{t-h / 2}^{t} x(s) d s\right. \\
& \left.+\frac{h}{2} \int_{t-h}^{t-h / 2} x(s) d s-\frac{3}{2} \int_{-h}^{-h / 2} \int_{t+\theta}^{t} x(s) d s d \theta\right) .
\end{aligned}
$$

For $h(t) \in[0, h / 2]$, the following formulas can be got using Lemmas 1 and 2:

$$
\begin{aligned}
& -\frac{h}{2} \int_{t-h / 2}^{t} \dot{x}^{T}(s) Q_{5} \dot{x}(s) d s=-\frac{h}{2} \\
& \cdot \int_{t-h / 2}^{t-h(t)} \dot{x}^{T}(s) Q_{5} \dot{x}(s) d s-\frac{h}{2} \\
& \cdot \int_{t-h(t)}^{t} \dot{x}^{T}(s) Q_{5} \dot{x}(s) d s \\
& \leq-\frac{h / 2}{h / 2-h(t)}\left(x(t-h(t))-x\left(t-\frac{h}{2}\right)\right)^{T} Q_{5} \\
& \cdot\left(x(t-h(t))-x\left(t-\frac{h}{2}\right)\right)-\frac{h / 2}{h(t)}(x(t) \\
& -x(t-h(t)))^{T} \cdot Q_{5}(x(t)-x(t-h(t))) \\
& -\frac{3 h / 2}{h / 2-h(t)}\left(x(t-h(t))+x\left(t-\frac{h}{2}\right)\right. \\
& \left.-\frac{h}{h / 2-h(t)} \int_{t-h / 2}^{t-h(t)} \dot{x}(s) d s\right)^{T} \cdot Q_{5}(x(t-h(t)) \\
& \left.+x\left(t-\frac{h}{2}\right)-\frac{h}{h / 2-h(t)} \int_{t-h / 2}^{t-h(t)} \dot{x}(s) d s\right) \\
& -\frac{3 h / 2}{h(t)}(x(t)+x(t-h(t)) \\
& \left.-\frac{h}{h(t)} \int_{t-h(t)}^{t} \dot{x}(s) d s\right)^{T} \cdot Q_{5}(x(t)+x(t-h(t)) \\
& \left.-\frac{h}{h(t)} \int_{t-h(t)}^{t} \dot{x}(s) d s\right) \\
& \leq-\frac{h / 2}{h / 2-h(t)}\left(x(t-h(t))-x\left(t-\frac{h}{2}\right)\right)^{T} Q_{5} \\
& \cdot\left(x(t-h(t))-x\left(t-\frac{h}{2}\right)\right)-\frac{h / 2}{h(t)}(x(t) \\
& -x(t-h(t)))^{T} \cdot Q_{5}(x(t)-x(t-h(t))) \\
& \leq-\left[\begin{array}{c}
x(t-h(t))-x\left(t-\frac{h}{2}\right) \\
x(t)-x(t-h(t))
\end{array}\right]^{T}\left[\begin{array}{cc}
Q_{5} & X_{1} \\
* & Q_{5}
\end{array}\right] \\
& {\left[\begin{array}{c}
x(t-h(t))-x\left(t-\frac{h}{2}\right) \\
x(t)-x(t-h(t))
\end{array}\right]}
\end{aligned}
$$

$$
\begin{aligned}
& -\frac{h}{2} \int_{t-h}^{t-h / 2} \dot{x}^{T}(s) Q_{6} \dot{x}(s) d s \leq-\left(x\left(t-\frac{h}{2}\right)\right. \\
& -x(t-h))^{T} Q_{6}\left(x\left(t-\frac{h}{2}\right)-x(t-h)\right) \\
& -3\left(x\left(t-\frac{h}{2}\right)+x(t-h)-\frac{4}{h} \int_{t-h}^{t-h / 2} x(s) d s\right)^{T} \\
& \cdot Q_{6}\left(x\left(t-\frac{h}{2}\right)+x(t-h)-\frac{4}{h} \int_{t-h}^{t-h / 2} x(s) d s\right) .
\end{aligned}
$$

At the same time, we can get the following inequation from formula (3):

$$
s_{i} f_{i}\left(\sigma_{i}(t)\right)\left[k_{i} h_{i}^{T} x(t)-f_{i}\left(\sigma_{i}(t)\right)\right] \geq 0
$$

It is equivalent to

$$
2\left[x^{T}(t) H K S f(\sigma(t))-f^{T}(\sigma(t)) S f(\sigma(t))\right] \geq 0,
$$

where

$$
\begin{aligned}
& S=\operatorname{diag}\left\{s_{1}, s_{2}, \ldots, s_{m}\right\}, \\
& K=\operatorname{diag}\left\{k_{1}, k_{2}, \ldots, k_{m}\right\} .
\end{aligned}
$$

According to inequalities (27), (28), (29), (30), and (32), we get

$\dot{V}(x(t))$

$$
\begin{aligned}
\leq & \sum_{i=1}^{5} \dot{V}_{i}(x(t)) \\
& +2\left[x^{T}(t) H K S f(\sigma(t))-f^{T}(\sigma(t)) S f(\sigma(t))\right] \\
\leq & \xi^{T}(t)\left(E+\Theta_{1}+\Psi_{1}+\eta^{T} \Omega \eta\right) \xi^{T}(t) .
\end{aligned}
$$

Obviously, LMI (25) with $r=1$ suggests that $\dot{V}(x(t))<0$ using Schur complement.

For $h(t) \in[h / 2, h]$, the following formulas can be got by using Lemmas 1 and 2:

$$
\begin{aligned}
-\frac{h}{2} \int_{t-h / 2}^{t} \dot{x}^{T}(s) Q_{5} \dot{x}(s) d s & \\
\leq & -\left(x(t)-x\left(t-\frac{h}{2}\right)\right)^{T} Q_{5}\left(x(t)-x\left(t-\frac{h}{2}\right)\right) \\
& -3\left(x(t)+x\left(t-\frac{h}{2}\right)-\frac{4}{h} \int_{t-h / 2}^{t} x(s) d s\right)^{T} \\
& \cdot Q_{5}\left(x(t)+x\left(t-\frac{h}{2}\right)-\frac{4}{h} \int_{t-h / 2}^{t} x(s) d s\right)
\end{aligned}
$$




$$
\begin{aligned}
- & \frac{h}{2} \int_{t-h}^{t-h / 2} \dot{x}^{T}(s) Q_{6} \dot{x}(s) d s \\
= & -\frac{h}{2} \int_{t-h}^{t-h(t)} \dot{x}^{T}(s) Q_{6} \dot{x}(s) d s \\
& -\frac{h}{2} \int_{t-h(t)}^{t-h / 2} \dot{x}^{T}(s) Q_{6} \dot{x}(s) d s \\
\leq- & {\left[\begin{array}{c}
x(t-h(t))-x(t-h) \\
x\left(t-\frac{h}{2}\right)-x(t-h(t))
\end{array}\right]\left[\begin{array}{ll}
Q_{6} & X_{2} \\
* & Q_{6}
\end{array}\right] } \\
& {\left[\begin{array}{c}
x(t-h(t))-x(t-h) \\
x\left(t-\frac{h}{2}\right)-x(t-h(t))
\end{array}\right] . }
\end{aligned}
$$

According to inequalities (27) and (28), (32), and (35), we have

$$
\begin{aligned}
& \dot{V}(x(t)) \\
& \leq \sum_{i=1}^{5} \dot{V}_{i}(x(t)) \\
& \quad+2\left[x^{T}(t) H K S f(\sigma(t))-f^{T}(\sigma(t)) S f(\sigma(t))\right] \\
& \quad \leq \xi^{T}(t)\left(E+\Theta_{1}+\Psi_{2}+\eta^{T} \Omega \eta\right) \xi^{T}(t) .
\end{aligned}
$$

Obviously, LMI (25) with $r=2$ suggests that $\dot{V}(x(t))<$ 0 using Schur complement. In conclusion, the asymptotic stability of nominal system (17) satisfying (3) has been proven.

Remark 11. If function $f(\sigma(t))$ of normal system (17) satisfies sector condition (4), for any $s_{i} \geq 0, i=1,2, \ldots, m$, it follows that

$$
s_{i} f_{i}\left(\sigma_{i}(t)\right) h_{i}^{T} x(t) \geq 0, \quad i=1,2, \ldots, m,
$$

which is equivalent to

$$
2 x^{T}(t) H S f(\sigma(t)) \geq 0 .
$$

Corollary 12. Nominal system (17) satisfying conditions (4) and (7) is asymptotically stable for given values $h \geq 0$, $h_{d}$, and $\tau_{d}<1$, if there exist appropriate dimensional matrices $X_{1}, X_{2}, X_{12}$, positive matrices $P=\left[P_{i j}\right]_{5 \times 5}, Q_{i}(i=$ $1,2, \ldots, 6), X_{11}, X_{22}$ positive semidefinite diagonal matrices $S=\operatorname{diag}\left\{s_{1}, s_{2}, \ldots, s_{m}\right\}$, and $\Lambda=\operatorname{diag}\left\{\lambda_{1}, \lambda_{2}, \ldots, \lambda_{m}\right\}$, such that the following LMIs hold:

$$
\widetilde{\Xi}_{r}=\left[\begin{array}{cc}
E+\Theta_{2}+\Psi_{r} & \eta^{T} \Omega \\
* & -\Omega
\end{array}\right]<0, \quad r=1,2
$$

as well as (22)-(24), where

$$
\Theta_{2}=2 e_{1}^{T} \mathrm{HSe}_{6} .
$$

Remark 13. Absolute stability criteria Theorem 10 and Corollary 12 proposed in this paper are less conservative than existing results which are shown by the following examples mentioned in the Section 4. The main reasons are the combination of introducing new inequality in Lemma 4 and the method of the exchange of integral order, based on the new constructed Lyapunov functionals.

The next theorem and corollary will investigate the robust stability for uncertain neutral-type Lur'e system (1) satisfying conditions (3)-(7) following similar methods of the proof for Theorem 10 and Corollary 12.

Theorem 14. System (1) satisfying conditions (3) and (5)-(7) is robustly absolutely stable for given values $h \geq 0, h_{d}, \tau_{d}<$ 1 , and $k_{l}>0(l=1,2, \ldots, m)$, if there exist appropriate dimensional matrices $X_{1}, X_{2}, X_{12}$, positive matrices $P=$ $\left[P_{i j}\right]_{5 \times 5}, Q_{i}(i=1,2, \ldots, 6), X_{11}, X_{22}$ positive semidefinite matrices $S=\operatorname{diag}\left\{s_{1}, s_{2}, \ldots, s_{m}\right\}, \Lambda=\operatorname{diag}\left\{\lambda_{1}, \lambda_{2}, \ldots, \lambda_{m}\right\}$, and scalars $\varepsilon_{r}>0$ such that the following LMIs hold:

$$
\left[\begin{array}{ccc}
E+\varepsilon_{r} \Delta E^{T} \Delta E+\Theta_{1}+\Psi_{r} & \eta^{T} \Omega & J \\
* & -\Omega & 0 \\
* & * & D^{T} \Omega D-\varepsilon_{r}
\end{array}\right]<0,
$$

as well as (22)-(24), where

$$
\begin{aligned}
\Delta E & =\left[\begin{array}{llllllllll}
E_{a} & E_{a 1} & 0 & 0 & 0 & E_{b} & 0 & 0 & 0 & 0
\end{array}\right] \\
J & =\left[\begin{array}{llllllllll}
J_{1}^{T} & J_{2}^{T} & 0 & 0 & J_{5}^{T} & J_{6}^{T} & J_{7}^{T} & J_{8}^{T} & J_{9}^{T} & J_{10}^{T}
\end{array}\right]^{T}, \\
J_{1} & =P_{11} D+A^{T} \Omega D, \\
J_{2} & =A_{1}^{T} \Omega D \\
J_{5} & =C^{T} \Omega D \\
J_{6} & =\Lambda H^{T} D+B^{T} \Omega D, \\
J_{7} & =P_{12}^{T} D \\
J_{8} & =P_{13}^{T} D \\
J_{9} & =P_{14}^{T} D, \\
J_{10} & =P_{15}^{T} D \\
r & =1,2 .
\end{aligned}
$$

Proof. If

$$
\begin{aligned}
& p(t) \\
& \quad=F(t)\left(E_{a} x(t)+E_{a 1} x(t-h(t))+E_{b} f(\sigma(t))\right),
\end{aligned}
$$

for any $\varepsilon>0$, we have the following inequality:

$$
\begin{aligned}
-\varepsilon p^{T}(t) p(t) & \\
& +\varepsilon\left(E_{a} x(t)+E_{a 1} x(t-h(t))+E_{b} f(\sigma(t))\right)^{T} \\
& \cdot\left(E_{a} x(t)+E_{a 1} x(t-h(t))+E_{b} f(\sigma(t))\right) \geq 0 .
\end{aligned}
$$


Using $A+D F(t) E_{a}, A_{1}+D F(t) E_{a 1}, B+D F(t) E_{b}$ instead of $A, A_{1}, B$ in formula (18), respectively, LMIs in (25) can be rewritten as (41).

Now, if function $f(\sigma(t))$ of system (1) satisfies sector condition (4), we have Corollary 15.

Corollary 15. System (1) satisfying conditions (4)-(7) is robustly absolutely stable for given values $h \geq 0, h_{d}$ and $\tau_{d}<1$, if there exist appropriate dimensional matrices $X_{1}, X_{2}, X_{12}$, positive matrices $P=\left[P_{i j}\right]_{5 \times 5}, Q_{i}(i=1,2, \ldots, 6), X_{11}, X_{22}$ positive semidefinite diagonal matrices

$$
\begin{gathered}
S=\operatorname{diag}\left\{s_{1}, s_{2}, \ldots, s_{m}\right\}, \\
\Lambda=\operatorname{diag}\left\{\lambda_{1}, \lambda_{2}, \ldots, \lambda_{m}\right\},
\end{gathered}
$$

and scalars $\varepsilon_{r}>0$ such that the following LMIs hold:

$$
\left[\begin{array}{ccc}
E+\varepsilon_{r} \Delta E^{T} \Delta E+\Theta_{2}+\Psi_{r} & \eta^{T} \Omega & J \\
* & -\Omega & 0 \\
* & * & D^{T} \Omega D-\varepsilon_{r}
\end{array}\right]<0,
$$

as well as (22)-(24), $r=1,2$.

Remark 16. Clearly, robust stability criteria Theorem 14 and Corollary 15 proposed in this paper would be less conservative, because of using the new inequality and some other techniques which are similar to Theorem 10 and Corollary 12.

Remark 17. In order to handle the integral term

$$
-\int_{-h_{2}}^{-h_{1}} \int_{t+\theta}^{t} \dot{x}^{T}(s) R \dot{x}(s) d s d \theta
$$

Jensen inequality [35] is always presented as

$$
\begin{aligned}
& -\int_{-h_{2}}^{0} \int_{t+\theta}^{t} \dot{x}^{T}(s) R \dot{x}(s) d s d \theta \\
& \leq-\frac{1}{h_{2}^{2}}\left(\int_{-h_{2}}^{0} \int_{t+\theta}^{t} \dot{x}(s) d s d \theta\right)^{T} \\
& \quad \cdot R\left(\int_{-h_{2}}^{0} \int_{t+\theta}^{t} \dot{x}(s) d s d \theta\right) .
\end{aligned}
$$

Obviously, Lemma 4 not only can obtain a more accurate bound than the Jensen inequality but also can reduce the number of decision variables. What is more, Lemma 4 can dispose the integral terms with $h_{1} \neq 0$. Therefore, Lemma 4 is used to derive our criterion, and the effectiveness of our method will be demonstrated by some existing numerical examples.

\section{Examples}

In this section, the effectiveness of our approaches will be shown by the following three numerical examples.
Example 1 (see [16, 19-21]). Consider Chua's circuit example discussed in [16, 19-21]. Chua's circuit is a simple nonlinear electronic circuit design. It can show the standard chaos theory. It was published by professor Leo Chua in 1983. The ease of making this circuit makes it a ubiquitous example of chaotic systems in the real world, leading somebody to declare it as a model of a chaotic system. The system equation is given by

$$
\begin{aligned}
& \dot{x}(t)=\alpha(y(t)-h(x(t))), \\
& \dot{y}(t)=x(t)-y(t)+z(t), \\
& \dot{z}(t)=-\beta y(t),
\end{aligned}
$$

with nonlinear characteristic

$$
\begin{aligned}
h(x(t))= & m_{1} x(t) \\
& +\frac{1}{2}\left(m_{0}-m_{1}\right)[|x(t)+c|-|x(t)-c|],
\end{aligned}
$$

and parameters $m_{0}=-1 / 7, m_{1}=2 / 7, \alpha=9, \beta=14.28$, and $c=1$. The system can be represented in normal Lur'e system framework (17) with

$$
\begin{aligned}
A & =\left[\begin{array}{ccc}
-\alpha m_{1}-1 & \alpha & 0 \\
1 & -2 & 1 \\
0 & -\beta & -1
\end{array}\right], \\
A_{1} & =\left[\begin{array}{ccc}
-6.0029 & 0 & 0 \\
-1.3367 & 0 & 0 \\
2.1264 & 0 & 0
\end{array}\right] \\
B & =\left[\begin{array}{r}
-\alpha\left(m_{0}-m_{1}\right) \\
0
\end{array}\right], \\
H & =\left[\begin{array}{l}
1 \\
0 \\
0
\end{array}\right], \\
C & =0 .
\end{aligned}
$$

The feedback nonlinear function belongs to $K_{[0,1]}$.

The MADBs of $h$ of system (49) for different $h_{d}$ have been shown in Table 1 with the existing results in [16, 19-21]. Table 1 shows us that the MADBs obtained by using Theorem 10 in this paper are better than the existing results in $[16,19-21]$.

Example 2 (see [12, 14-20]). Consider the following nominal neutral-type Lur'e systems:

$$
\begin{aligned}
\dot{x}(t)-C \dot{x}(t-\tau(t))= & A x(t)+A_{1} x(t-h(t)) \\
& +B f(\sigma(t)), \\
\sigma(t)= & H^{T} x(t), \quad \forall t \geq 0,
\end{aligned}
$$


TABLE 1: MADBs $h$ for different $h_{d}$ (Example 1).

\begin{tabular}{lccccc}
\hline$h_{d}$ & 0 & 0.3 & 0.6 & 0.9 & $>1.0$ \\
\hline$[21]$ & 0.1622 & 0.1591 & 0.1566 & 0.1541 & 0.1527 \\
{$[16]$} & 0.1745 & 0.1698 & 0.1698 & 0.1698 & 0.1698 \\
{$[19]$} & 0.1747 & 0.1710 & 0.1703 & 0.1703 & 0.1703 \\
{$[20]$} & 0.1771 & 0.1721 & 0.1715 & 0.1715 & 0.1715 \\
Theorem 10 & 0.2790 & 0.2691 & 0.2553 & 0.2463 & 0.2463 \\
\hline
\end{tabular}

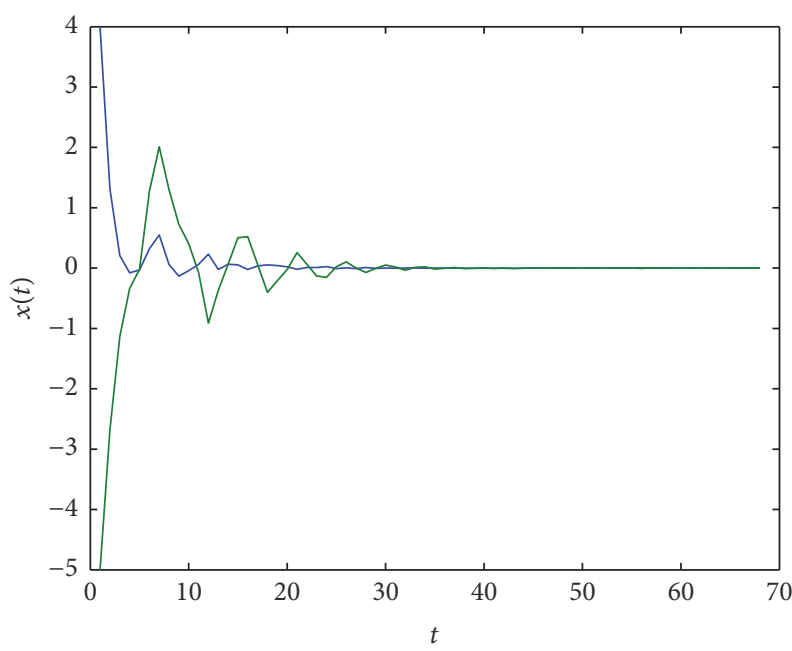

FIgURE 2: The dynamical behavior for Example 2.

with

$$
\begin{aligned}
A & =\left[\begin{array}{cc}
-2 & 0.5 \\
0 & -1
\end{array}\right], \\
A_{1} & =\left[\begin{array}{cc}
1 & 0.4 \\
0.4 & -1
\end{array}\right], \\
B & =\left[\begin{array}{l}
-0.5 \\
-0.75
\end{array}\right], \\
C & =\left[\begin{array}{ll}
0.2 & 0.1 \\
0.1 & 0.2
\end{array}\right], \\
H & =\left[\begin{array}{l}
0.2 \\
0.6
\end{array}\right] .
\end{aligned}
$$

The maximum allowable delay bounds (MADBs) of $h$ of nominal neutral-type Lur'e system (52) for different $h_{d}$ and $\tau_{d}$ have been shown in Table 2 along with the existing results in $[12,14-20]$. Applying Matlab LMI-toolbox, one can get the simulation result in Figure 1. Table 2 shows us that the MADBs obtained by using Corollary 15 in this paper are better than the existing results in $[12,14-20]$. What is more,

\begin{tabular}{|c|c|c|c|c|c|}
\hline$\tau_{d}$ & $h_{d}$ & 0.2 & 0.4 & 0.6 & 0.8 \\
\hline \multirow{9}{*}{0.1} & {$[12]$} & 2.2424 & 1.5434 & 0.9888 & 0.7228 \\
\hline & {$[14]$} & 2.6062 & 1.9730 & 1.6408 & 1.1854 \\
\hline & {$[15]$} & 2.6488 & 2.0055 & 1.6679 & 1.2049 \\
\hline & [17] & 2.9962 & 2.1316 & 1.7138 & 1.3204 \\
\hline & {$[16]$} & 2.9997 & 2.1012 & 1.7307 & 1.3359 \\
\hline & {$[18]$} & 2.6052 & 1.9538 & 1.6232 & 1.1659 \\
\hline & [19] & 3.0717 & 2.1171 & 1.7310 & 1.3367 \\
\hline & [20] & 3.1743 & 2.1789 & 1.7467 & 1.7153 \\
\hline & Corollary 15 & 3.4880 & 2.3787 & 1.8062 & 1.4625 \\
\hline \multirow{8}{*}{0.5} & [12] & 1.8344 & 1.3029 & 0.7793 & 0.6282 \\
\hline & [14] & 2.1537 & 1.7041 & 1.3828 & 1.0095 \\
\hline & {$[15]$} & 2.1883 & 1.7322 & 1.4056 & 1.0262 \\
\hline & [17] & 2.4472 & 1.8289 & 1.4551 & 1.1058 \\
\hline & {$[16]$} & 2.4563 & 1.8009 & 1.4823 & 1.1157 \\
\hline & {$[18]$} & 2.1532 & 1.6986 & 1.3687 & 1.0066 \\
\hline & [19] & 2.5131 & 1.8146 & 1.4951 & 1.1168 \\
\hline & Corollary 15 & 2.8538 & 2.0236 & 1.5679 & 1.2425 \\
\hline \multirow{8}{*}{0.9} & [12] & 0.1085 & 0.1019 & 0.0983 & 0.0967 \\
\hline & {$[14]$} & 0.1122 & 0.1088 & 0.1086 & 0.1086 \\
\hline & [15] & 0.1122 & 0.1089 & 0.1086 & 0.1086 \\
\hline & [17] & 0.1126 & 0.1102 & 0.1102 & 0.1102 \\
\hline & [16] & 0.1130 & 0.1105 & 0.1105 & 0.1105 \\
\hline & {$[18]$} & 0.1221 & 0.1087 & 0.1083 & 0.1083 \\
\hline & [19] & 0.1227 & 0.1197 & 0.1197 & 0.1197 \\
\hline & Corollary 15 & 0.1799 & 0.1697 & 0.1660 & 0.1658 \\
\hline
\end{tabular}
for all the nonlinear function $f(\sigma(t)) \in R^{m}$ with condition (3) or condition (4), the simulation study is entirely viable, which has been shown in this example (Figure 2).
TABLE 2: MADBs $h$ for different $h_{d}$ and $\tau_{d}$ (Example 2).

Example 3 (see $[15-17,19,20]$ ). Consider uncertain system (1) with the following parameters:

$$
\begin{aligned}
A & =\left[\begin{array}{cc}
-2 & 0 \\
0 & -0.9
\end{array}\right], \\
A_{1} & =\left[\begin{array}{cc}
-1 & 0 \\
-1 & -1
\end{array}\right], \\
B & =\left[\begin{array}{c}
-0.2 \\
-0.3
\end{array}\right], \\
C & =\left[\begin{array}{ll}
0 & 0 \\
0 & 0
\end{array}\right], \\
H & =\left[\begin{array}{l}
0.6 \\
0.8
\end{array}\right], \\
D & =E_{a}=E_{a 1}=\left[\begin{array}{cc}
0.1 & 0 \\
0 & 0.1
\end{array}\right],
\end{aligned}
$$


TABLE 3: MADBs $h$ for different $K$ (Example 3).

\begin{tabular}{lcc}
\hline$K$ & Methods & $h$ \\
\hline & {$[15]$} & 4.3196 \\
& {$[17]$} & 5.1366 \\
$f(\cdot) \in K_{[0,0.5]}$ & {$[16]$} & 5.1666 \\
& {$[19]$} & 5.3486 \\
& {$[20]$} & 5.4972 \\
& Theorem 14 & 5.5513 \\
\hline & {$[15]$} & 4.1792 \\
$f(\cdot) \in K_{[0,100]}$ & {$[17]$} & 4.9572 \\
& {$[16]$} & 4.9731 \\
& {$[19]$} & 5.1828 \\
& Theorem 14 & 5.3557 \\
\hline & {$[15]$} & 4.1581 \\
$f(\cdot) \in K_{[0, \infty)}$ & {$[17]$} & 4.9304 \\
& {$[16]$} & 4.9431 \\
& {$[19]$} & 5.1654 \\
& {$[20]$} & 5.2489 \\
& Corollary 15 & 5.3186 \\
\hline
\end{tabular}

$$
\begin{aligned}
& E_{b}=\left[\begin{array}{c}
0.1 \\
0
\end{array}\right], \\
& E_{c}=\left[\begin{array}{cc}
\sin (\omega(t)) & 0 \\
0 & \sin (\omega(t))
\end{array}\right] .
\end{aligned}
$$

The MADBs of $h$ with $h_{d}=0$ and $\tau_{d}<1$ for different values of $K_{\left[0, k_{i}\right]}$ are listed in Table 3 by using Theorem 14 and Corollary 15 along with the existing results in [15-17, 19, 20]. Table 3 shows us that the MADBs obtained by using Theorem 14 and Corollary 15 in this paper are better than the existing results in $[15-17,19,20]$.

\section{Conclusions}

In this paper, we have studied the stability for a class of neutral-type Lur'e systems with time-varying delays and sector bounded nonlinearities by using a new LKF. The LKF contains not only double-integral terms but also tripleintegral terms. Using some effective techniques, such as a novel integral inequality, a piecewise analysis method, and the reciprocally convex combination inequality, instead of the general free-weighting matrix method, the delaydependent stability criteria derived in the form of LMIs are less conservative than some existing results. The effectiveness and the less conservatism of stability criteria proposed in this paper are demonstrated by using standard numerical examples.

\section{Competing Interests}

The authors declare that they have no competing interests.

\section{Acknowledgments}

This work is supported by National Natural Science Foundation of China (Grant nos. 11461082, 11372005, and 11601474), the Specialized Research Fund For the Doctoral Program of Higher Education (Grant no. 135578), and the Scientific Research Fund Project in Yunnan Provincial Department of Education (Grant no. 2015J069).

\section{References}

[1] X. Liao, Absolute Stability of Nonlinear Control Systems, Science Press, Beijing, China, 1993.

[2] E. Tian, D. Yue, and C. Peng, "Delay-fractioning-dependent robust $\mathrm{H}_{\infty}$ control for linear systems with delay in state or input and its applications," International Journal of Systems Science, vol. 43, no. 5, pp. 820-833, 2012.

[3] H. Zhang and Z. Liu, "Stability analysis for linear delayed systems via an optimally dividing delay interval approach," Automatica, vol. 47, no. 9, pp. 2126-2129, 2011.

[4] N. S. Ahmad, W. P. Heath, and G. Li, "LMI-based stability criteria for discrete-time Lur'e systems with monotonic, sectorand slope-restricted nonlinearities," Institute of Electrical and Electronics Engineers. Transactions on Automatic Control, vol. 58, no. 2, pp. 459-465, 2013.

[5] G. Rajchakit, T. Rojsiraphisal, and M. Rajchakit, "Robust stability and stabilization of uncertain switched discrete-time systems," Advances in Difference Equations, vol. 2012, no. 1, article 134, 15 pages, 2012.

[6] J. Wang, Z. Duan, Y. Yang, and L. Huang, Analysis and Control of Nonlinear Systems with Stationary Sets, World Scientific, Singapore, 2009.

[7] A. Lurie, Some nonlinear problem in the theory of automatic control, [phd thesis], Her Majesty's Stationery Office, London, UK, 1957.

[8] F. Qiu, B. Cui, and Y. Ji, "Delay-dividing approach for absolute stability of Lurie control system with mixed delays," Nonlinear Analysis. Real World Applications. An International Multidisciplinary Journal, vol. 11, no. 4, pp. 3110-3120, 2010.

[9] P. Park, "Stability criteria of sector- and slope-restricted Lur'e systems," IEEE Transactions on Automatic Control, vol. 47, no. 2, pp. 308-313, 2002.

[10] G. Rajchakit, "Robust stability and stabilization of nonlinear uncertain stochastic switched discrete-time systems with interval time-varying delays," Applied Mathematics \& Information Sciences, vol. 6, no. 3, pp. 555-565, 2012.

[11] G. Rajchakit, "Switching design for the asymptotic stability and stabilization of nonlinear uncertain stochastic discretetime systems," International Journal of Nonlinear Sciences and Numerical Simulation, vol. 14, no. 1, pp. 33-44, 2013.

[12] J. Gao, H. Su, X. Ji, and J. Chu, "Stability analysis for a class of neutral systems with mixed delays and sector-bounded nonlinearity," Nonlinear Analysis: Real World Applications, vol. 9, no. 5, pp. 2350-2360, 2008.

[13] C. Yin, S.-m. Zhong, and W.-f. Chen, "On delay-dependent robust stability of a class of uncertain mixed neutral and Lur'e dynamical systems with interval time-varying delays," Journal of the Franklin Institute. Engineering and Applied Mathematics, vol. 347, no. 9, pp. 1623-1642, 2010.

[14] Y. Wang, X. Zhang, and Y. He, "Improved delay-dependent robust stability criteria for a class of uncertain mixed neutral 
and Lur'e dynamical systems with interval time-varying delays and sector-bounded nonlinearity," Nonlinear Analysis: Real World Applications, vol. 13, no. 5, pp. 2188-2194, 2012.

[15] K. Ramakrishnan and G. Ray, "Improved delay-rangedependent robust stability criteria for a class of Lur'e systems with sector-bounded nonlinearity," Journal of the Franklin Institute, vol. 348, no. 8, pp. 1769-1786, 2011.

[16] K. Ramakrishnan and G. Ray, "An improved delay-dependent stability criterion for a class of lur'e systems of neutral type," Journal of Dynamic Systems, Measurement and Control, Transactions of the ASME, vol. 134, no. 1, 2012.

[17] W. Duan, B. Du, J. You, and Y. Zou, "Improved robust stability criteria for a class of Lur'e systems with interval time-varying delays and sector-bounded nonlinearity," International Journal of Systems Science, vol. 46, no. 5, pp. 944-954, 2015.

[18] K. Shi, H. Zhu, S. Zhong, Y. Zeng, and Y. Zhang, "Improved delay-dependent robust stability criteria for a class of uncertain neutral type Lur'e systems with discrete and distributed delays," Mathematical Problems in Engineering, vol. 2014, Article ID 980351, 14 pages, 2014.

[19] W. Duan, B. Du, Z. Liu, and Y. Zou, "Improved stability criteria for uncertain neutral-type Lur'e systems with time-varying delays," Journal of the Franklin Institute, vol. 351, no. 9, pp. 45384554, 2014.

[20] Y. Wang, Y. Xue, and X. Zhang, "Less conservative robust absolute stability criteria for uncertain neutral-type Lure systems with time-varying delays," Journal of the Franklin Institute. Engineering and Applied Mathematics, vol. 353, no. 4, pp. 816833, 2016.

[21] Q.-L. Han, "On designing time-varying delay feedback controllers for master-slave synchronization of Lur'e systems," IEEE Transactions on Circuits and Systems I: Regular Papers, vol. 54, no. 7, pp. 1573-1583, 2007.

[22] C. Yang, Q. Zhang, J. Sun, and T. Chai, "Lur'e Lyapunov function and absolute stability criterion for Lur'e singularly perturbed systems," IEEE Transactions on Automatic Control, vol. 56, no. 11, pp. 2666-2671, 2011.

[23] W.-Y. Duan, C. Cai, and Y. Zou, "Synchronization for Lurie type complex dynamical networks with time-varying delay based on linear feedback controller," in Proceedings of the 10th World Congress on Intelligent Control and Automation (WCICA '12), pp. 1389-1394, Beijing, China, July 2012.

[24] G. Rajchakit, "Synchronization criteria for singular complex dynamical networks with delayed coupling and non-delayed coupling," Journal of Computational Analysis and Applications, vol. 16, no. 1, p. 1019, 2016.

[25] W. Duan and C. Cai, "Delay-range-dependent stability criteria for delayed discrete-time Lur'e system with sector-bounded nonlinearities," Nonlinear Dynamics, vol. 78, no. 1, pp. 135-145, 2014.

[26] M. Rajchakit, P. Niamsup, T. Rojsiraphisal, and G. Rajchakit, "Delay-dependent guaranteed cost controller design for uncertain neural networks with interval time-varying delay," Abstract and Applied Analysis, vol. 2012, Article ID 587426, 16 pages, 2012.

[27] Q.-L. Han, A. Xue, S. Liu, and X. Yu, "Robust absolute stability criteria for uncertain Lure systems of neutral type," International Journal of Robust and Nonlinear Control, vol. 18, no. 3, pp. 278-295, 2008.

[28] K.-W. Yu and C.-H. Lien, "Stability criteria for uncertain neutral systems with interval time-varying delays," Chaos, Solitons \& Fractals, vol. 38, no. 3, pp. 650-657, 2008.
[29] O. M. Kwon, J. H. Park, and S. M. Lee, "An improved delay-dependent criterion for asymptotic stability of uncertain dynamic systems with time-varying delays," Journal of Optimization Theory and Applications, vol. 145, no. 2, pp. 343-353, 2010.

[30] O. Kwon, J. Park, and S. Lee, "Corrigendum to published papers in Applied Mathematics and Computation," Applied Mathematics and Computation, vol. 215, no. 1, pp. 427-430, 2009.

[31] A. Seuret and F. Gouaisbaut, "Integral inequality for timevarying delay systems," in Proceedings of the 12th European Control Conference (ECC '13), pp. 3360-3365, IEEE, Zurich, Switzerland, July 2013.

[32] J.-F. Gao, H.-Y. Su, X.-F. Ji, and J. Chu, "New delay-dependent absolute stability criteria for Lurie control systems," Acta Automatica Sinica, vol. 34, no. 10, pp. 1275-1280, 2008.

[33] D. Yue and Q.-L. Han, "A delay-dependent stability criterion of neutral systems and its application to a partial element equivalent circuit model," IEEE Transactions on Circuits and Systems II: Express Briefs, vol. 51, no. 12, pp. 685-689, 2004.

[34] P. Park, J. W. Ko, and C. Jeong, "Reciprocally convex approach to stability of systems with time-varying delays," Automatica. A Journal of IFAC, the International Federation of Automatic Control, vol. 47, no. 1, pp. 235-238, 2011.

[35] J. Sun, G. P. Liu, and J. Chen, "Delay-dependent stability and stabilization of neutral time-delay systems," International Journal of Robust and Nonlinear Control, vol. 19, no. 12, pp. 13641375, 2009.

[36] M. Park, O. Kwon, J. H. Park, S. Lee, and E. Cha, "Stability of time-delay systems via Wirtinger-based double integral inequality," Automatica. A Journal of IFAC, the International Federation of Automatic Control, vol. 55, pp. 204-208, 2015.

[37] P. Park, W. I. Lee, and S. Y. Lee, "Auxiliary function-based integral inequalities for quadratic functions and their applications to time-delay systems," Journal of the Franklin Institute. Engineering and Applied Mathematics, vol. 352, no. 4, pp. 13781396, 2015. 


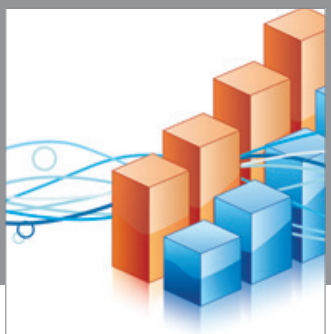

Advances in

Operations Research

vatem alat4

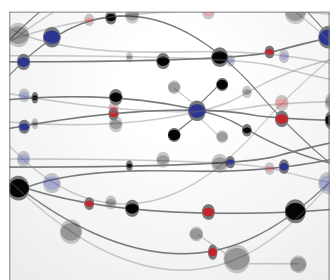

\section{The Scientific} World Journal
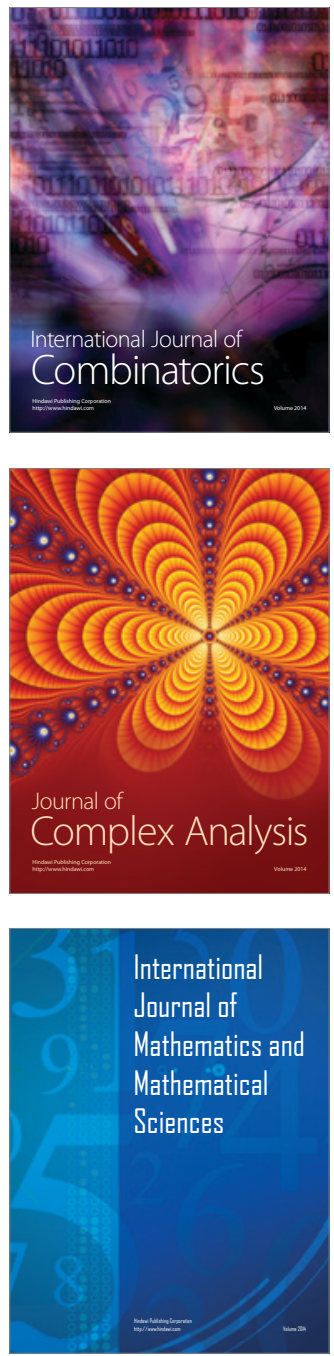
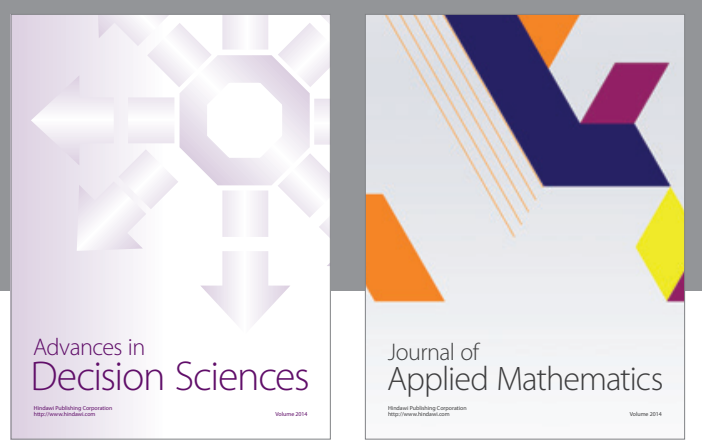

Algebra

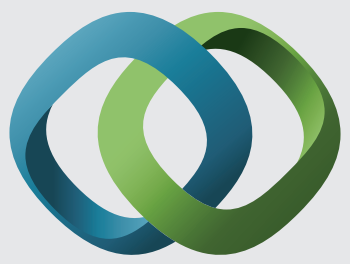

\section{Hindawi}

Submit your manuscripts at

https://www.hindawi.com
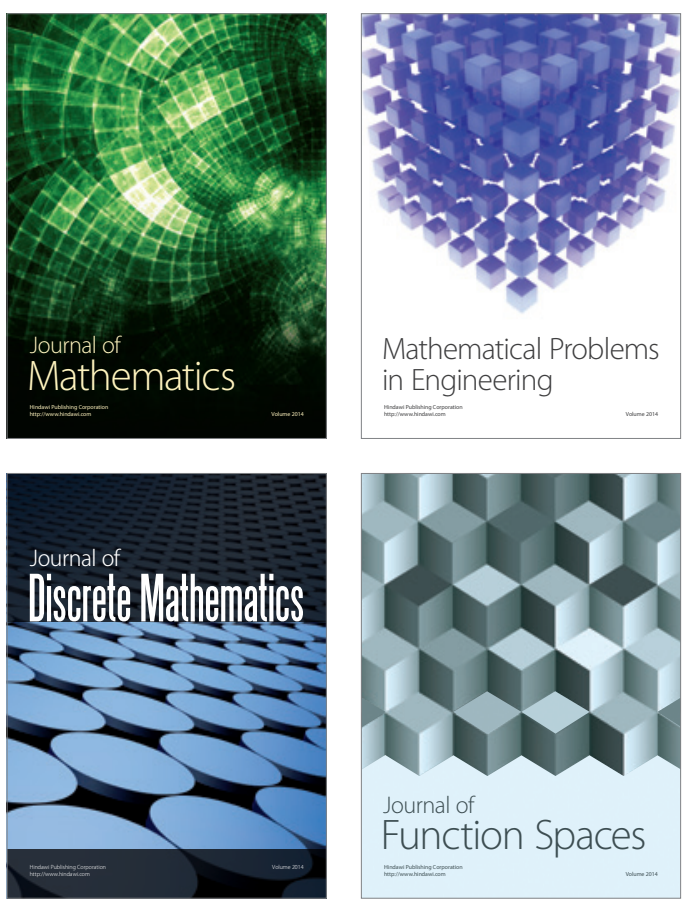

Mathematical Problems in Engineering
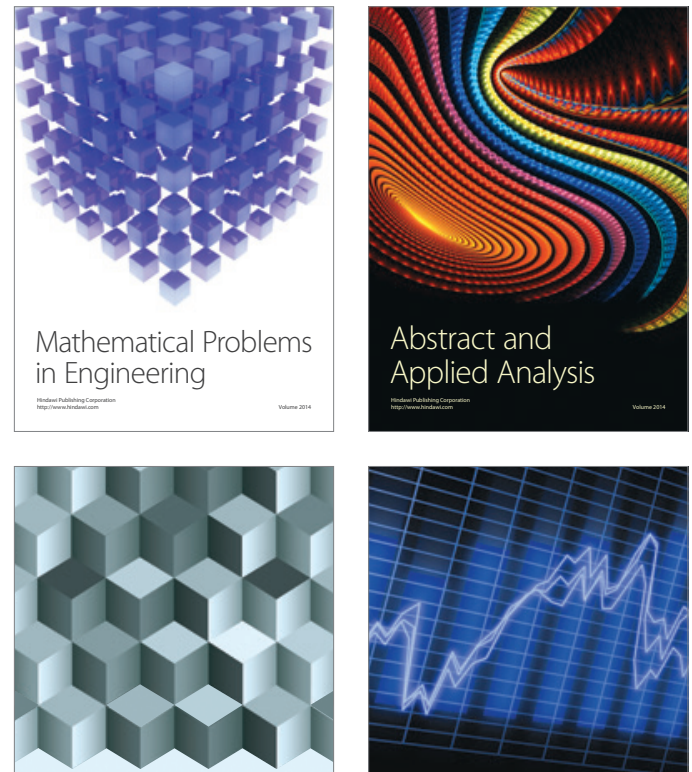

Journal of

Function Spaces

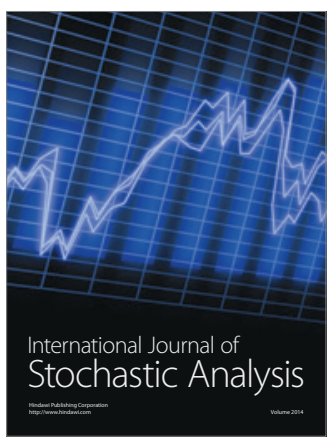

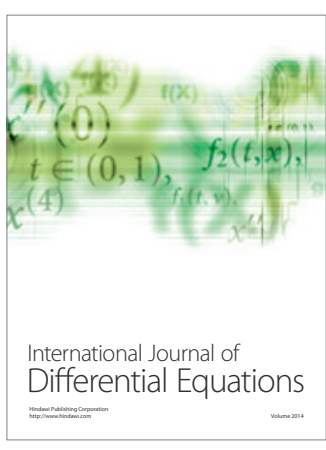
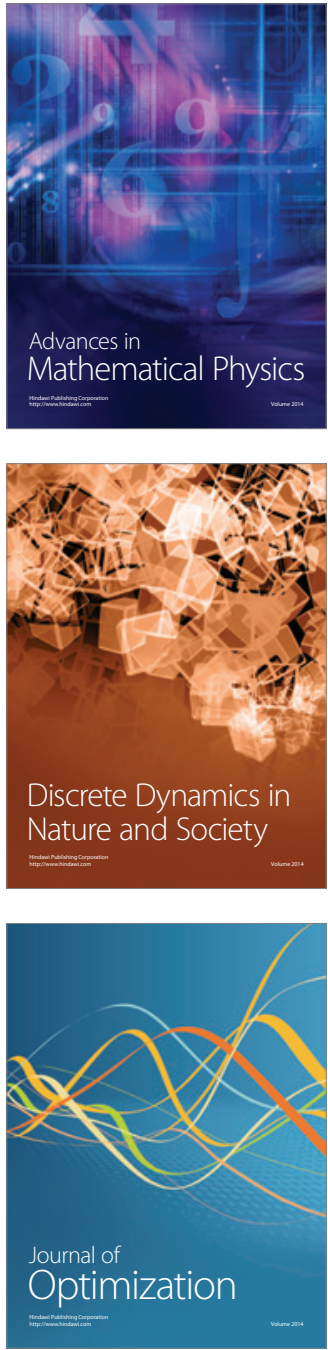